\title{
LINC01638 IncRNA promotes cancer cell proliferation in hepatocellular carcinoma by increasing cancer cell glucose uptake
}

\author{
XIAOLI CHEN ${ }^{*}$, LILI WANG ${ }^{*}$ and HUI WANG \\ Ten Areas of Liver Disease, Sixth People's Hospital of Qingdao, Qingdao, Shandong 266000, P.R. China
}

Received November 12, 2018; Accepted June 13, 2019

DOI: $10.3892 / 01.2019 .10682$

\begin{abstract}
The aim of the present study was to examine the function of long intergenic non-protein coding RNA 1638 (LINC01638) long non-coding RNA (lncRNA) in hepatocellular carcinoma (HCC). In the present study, gene expression was analyzed using qPCR and western blotting. Glucose uptake was analyzed using a glucose uptake assay and cell proliferation was analyzed using a cell counting kit-8 assay. LINC01638 1ncRNA and glucose transporter 1 (GLUT1) were upregulated in tumor tissues compared with adjacent healthy tissues of patients with HCC. Expression levels of LINC01638 lncRNA and GLUT1 were positively correlated only in tumor tissues; however, there was no correlation in adjacent healthy tissues. Overexpression of LINC01638 lncRNA and GLUT1 promoted glucose uptake, while LINC01638 lncRNA and GLUT1-knockdown led to inhibited glucose uptake of cells of HCC cell lines. Overexpression of LINC01638 lncRNA mediated the upregulation of GLUT1 expression and accelerated cell proliferation. GLUT1 overexpression failed to significantly affect LINC01638 IncRNA expression, however also promoted cancer cell proliferation. In addition, GLUT1-knockdown attenuated the effects of LINC01638 overexpression on cancer cell proliferation. Therefore, LINC01638 IncRNA promoted cancer cell proliferation in HCC, potentially by increasing cancer cell glucose uptake.
\end{abstract}

\section{Introduction}

Liver cancer is one of the most frequently diagnosed human malignancies and is also one of the leading causes

Correspondence to: Dr Hui Wang, Ten Areas of Liver Disease, Sixth People's Hospital of Qingdao, 9 Fushun Road, Qingdao, Shandong 266000, P.R. China

E-mail:vp72332@163.com

\section{*Contributed equally}

Key words: hepatocellular carcinoma, long intergenic non-protein coding RNA 1638, long non-coding RNA, glucose transporter 1, glucose uptake of cancer-associated mortality worldwide (1). As risk factor screening programs have become more popular, liver cancer incidence rates have declined over the past decade in a number of Asian countries (2). However, the incidence of liver in the majority of developed countries is increasing (2). In spite of efforts made on the treatment of liver cancer (3), survival of patients with liver cancer remains poor $(4,5)$, largely due to the high prevalence of cancer metastasis by the time of diagnosis before radical treatment (6).

Cancer cells are characterized by accelerated energy metabolism compared with normal cells (7). In effect, inhibition of energy metabolism is considered to be a promising target for cancer treatment (8). Glucose transporter 1 (GLUT1) is a solute carrier that facilitates the transport of glucose across mammalian cell plasma membrane (9). A growing body of literatures has indicated that GLUT1 also contributes to the development of different types of cancer, partially through the interactions with long non-coding RNAs (lncRNAs) $(10,11)$, which are a subgroup of non-coding RNAs with critical roles in cancer biology (12). It is has been previously reported that long intergenic non-protein coding RNA 1638 (LINC01638) promotes breast cancer (13), while its roles in other types of cancers remain unclear. The present study indicated that LINC01638 lncRNA promoted cancer cell proliferation in hepatocellular carcinoma (HCC), a major type of liver cancer, potentially by increasing cancer cell glucose uptake and promoting GLUT1 expression.

\section{Materials and methods}

Patients, human tissues and HCC cell lines. A total of 74 patients with HCC admitted to and treated at the Sixth People's Hospital of Qingdao (Qindao, China) between May 2016 and 2018 were selected as research subjects. Inclusion criteria were as follows: HCC diagnosed by pathological examinations; patients with normal function of other major organs; patients with a complete medical record and patients, who had understood the experimental protocol. Exclusion criteria were as follows: Patients combined with other diseases, including chronic diseases other than liver diseases; patients who received treatment within 3 months prior to admission. Liver biopsy was performed on all patients, and tumor tissues as well as adjacent healthy tissues, were collected. Tissues were stored in liquid nitrogen prior to use. The 74 patients 
included 40 males and 34 females, and age ranged between 36 and 66 years (mean age, $49.1 \pm 5.6$ years). There were 8 cases of stage I, 18 cases of stage II, 30 cases of stage III and 18 cases of stage IV according to the American Joint Committee on Cancer staging system (14). The 74 patients included $29 \mathrm{HBV}$-positive cases, $17 \mathrm{HCV}$-positive cases, 12 both-positive cases and 16 both-negative cases. This study was approved by the ethics committee of Sixth People's Hospital of Qingdao (Qingdao, China). Written informed consent was obtained from all patients.

The present study included two HCC cell lines, SNU-398 and SNU-182. The cell lines and RPMI-1640 medium were purchased from American Type Culture Collection (ATCC). Cells were cultured in RPMI-1640 medium supplemented with $10 \%$ heat-inactivated FBS (ATCC) in an incubator at $37^{\circ} \mathrm{C}$ with $5 \% \mathrm{CO}_{2}$.

Reverse transcription-quantitative PCR (RT-qPCR). To determine the expression of LINC01638 lncRNA and GLUT1, total RNA was extracted using TRIzol reagent (Thermo Fisher Scientific, Inc.), RT was performed using Applied Biosystems $^{\mathrm{TM}}$ High-Capacity cDNA Reverse Transcription kit (Applied Biosystems; Thermo Fisher Scientific, Inc.) and the reverse transcription protocol was $20 \mathrm{~min}$ at $55^{\circ} \mathrm{C}$ and $10 \mathrm{~min}$ at $80^{\circ} \mathrm{C}, \mathrm{PCR}$ reaction systems were prepared using a SYBR ${ }^{\circledR}$ Green Quantitative RT-qPCR kit (Sigma-Aldrich; Merck KGaA). Primers of LINC01638 1ncRNA, GLUT1 and $\beta$-actin endogenous control were designed and synthesized by Sangon Biotech Co., Ltd. Primer sequences were as follows: 5'-CCTCTGGAATACATCAGCAC-3' (forward) and 5'-GGT GGAGACTGTAGTGAGCC-3' (reverse) for LINC01638; 5'-AGGTGATCGAGGAGTTCTAC-3' (forward) and 5'-TCA AAGGACTTGCCCAGTTT-3' (reverse) for GLUT1; 5'-GAG ACCTTCAACACCCCAGCC-3' (forward) and 5'-AATGTC ACGCACGATTTCCC-3' (reverse) for $\beta$-actin. All PCR reactions were performed on an $\mathrm{S}_{1000}{ }^{\mathrm{TM}}$ Thermal Cycler (Bio-Rad Laboratories Inc.). The thermocycling conditions were: $95^{\circ} \mathrm{C}$ for $50 \mathrm{sec}$, followed by 40 cycles of $95^{\circ} \mathrm{C}$ for $10 \mathrm{sec}$ and $55^{\circ} \mathrm{C}$ for $40 \mathrm{sec}$. Expression of LINC01638 lncRNA and GLUT1 was normalized to $\beta$-actin endogenous control using $2^{-\Delta \Delta \mathrm{Cq}}$ method (15). Similar expression results were obtained using 18S rRNA as the endogenous control (data not shown).

Cell transfection. LINC01638 lncRNA and GLUT1 expressing vectors (pcDNA3.1) and empty vectors were designed and constructed by Sangon Biotech Co., Ltd. GLUT1 small interfering RNA (siRNA; 5'-CCAAGAGTGTGCTAAAGA ATT-3'), LINC01638 siRNA (5'-CATACATACAACTCC AAAAAGT-3'), as well as negative control siRNA (5'-ACA ATGAGTCGTAGCATGG-3') were designed and synthesized by Shanghai GenePharma Co., Ltd. SNU-398 and SNU-182 cell lines were cultured overnight to reach $70-80 \%$ confluency and all cell transfections were performed using Lipofectamine ${ }^{\circledR} 2000$ (cat. no. 11668-019; Invitrogen; Thermo Fisher Scientific Inc.). All operations were performed in strict accordance with the manufacturer's protocols. Doses of vectors and siRNAs were 10 and $35 \mathrm{nM}$, respectively. Cells only treated with Lipofectamine ${ }^{\circledR} 2000$ were the control cells. Empty vector or negative control siRNA transfection was the negative control. Transfection efficacy was determined by RT-qPCR. In cases of co-transfection, expression vector $(10 \mathrm{nM})$ and siRNA $(35 \mathrm{nM})$ were mixed with cells at the same time.

Glucose uptake assay. LINC01638 lncRNA and GLUT1 expression was detected by RT-qPCR at $24 \mathrm{~h}$ after transfection. Cells were harvested for glucose uptake assay only when GLUT1/LINC01638 overexpression was $>200 \%$ and knockdown had reached 50\%. Cells of both SNU-398 and SNU-182 cell lines were harvested and washed with PBS buffer. A total of $1 \times 10^{6}$ cells were subsequently dissolved in Krebs-Ringer-HEPES (KRH) buffer (25 mM HEPES pH 7.4, $1.3 \mathrm{mM} \mathrm{KH}_{2} \mathrm{PO}_{4}, 120 \mathrm{mM} \mathrm{NaCl}$, $1.2 \mathrm{mM} \mathrm{MgSO}_{4}, 1.3 \mathrm{mM} \mathrm{CaCl}_{2}$ and $5 \mathrm{mM} \mathrm{KCl}$ ). A total of $1 \mu \mathrm{Ci}$ of $(3 \mathrm{H})$-2-deoxyglucose was added and cells were incubated at $37^{\circ} \mathrm{C}$ for $30 \mathrm{~min}$ to initiate glucose uptake. Cells were washed with ice-cold KRH buffer to stop glucose uptake. Finally, cells were incubated with lysis buffer (10 mM Tris- $\mathrm{HCl} \mathrm{pH}$ 8.0, 0.2\% SDS) to induce cell lysis, and radioactivity was measured using liquid scintillation spectrometry (LS6500 Liquid Scintillation Counter; Beckman Coulter, Inc.). Disintegrations per minute was used to represent glucose uptake.

Cell proliferation assay. LINC01638 1ncRNA and GLUT1 expression was detected by RT-qPCR at $24 \mathrm{~h}$ after transfection. Cells were harvested for cell proliferation analysis only in cases of LINC01638 lncRNA, and when GLUT1 overexpression rate was $>200 \%$ and knockdown rate had reached $50 \%$. In brief, cells were harvested, and single cell suspensions were prepared. Cell density was adjusted to $4 \times 10^{4}$ cells $/ \mathrm{ml}$. Cell suspensions were then transferred to a 96 -well plate, $0.1 \mathrm{ml}$ per well. Cells were cultivated in an incubator at $37^{\circ} \mathrm{C}$ with $5 \% \mathrm{CO}_{2}$, followed by the addition of Cell Counting Kit-8 solution (10 $\mu \mathrm{l}$; Sigma-Aldrich; Merck KGaA) 24, 48, 72 and 96 h later. Cells were subsequently cultivated for an additional $4 \mathrm{~h}$, and optical density values were measured at a wavelength of $450 \mathrm{~nm}$.

Western blot analysis. LINC01638 lncRNA and GLUT1 expression was detected by RT-qPCR at $24 \mathrm{~h}$ following transfection. Cells were harvested for western blot analysis only when GLUT1/LINC01638 overexpression was $>200 \%$ and knockdown rate had reached $50 \%$. To detect the expression of GLUT1, total protein was extracted using RIPA buffer (Thermo Fisher Scientific, Inc.). Protein concentrations were measured using a bicinchoninic acid assay (Thermo Fisher Scientific, Inc.). Following denaturation, protein samples $(30 \mu \mathrm{g}$ per well) were separated by SDS-PAGE on $12 \%$ gels, followed by gel transfer to polyvinylidene difluoride membranes. Membranes were blocked in 5\% non-fat milk for $2 \mathrm{~h}$ at room temperature, followed by incubation with primary antibodies at $4^{\circ} \mathrm{C}$ for $18 \mathrm{~h}$ : Rabbit anti-human GLUT1 (dilution, 1:1,400; cat. no. ab32551; Abcam) and GAPDH (dilution, 1:1,400; cat. no. ab8245; Abcam). Cells were further incubated with goat anti-rabbit horseradish peroxidase (IgG H\&L; cat. no. ab6721; Abcam) for $2 \mathrm{~h}$ at $24^{\circ} \mathrm{C}$. Enhanced chemiluminescence detection reagent (Sigma-Aldrich; Merck KGaA) was used to develop signals. Signals were normalized using Image J 1.8.0 software (National Institutes of Health).

Statisticalanalysis.Allexperiments in this study were performed in triplicate (biological replicates) and mean \pm standard 
A

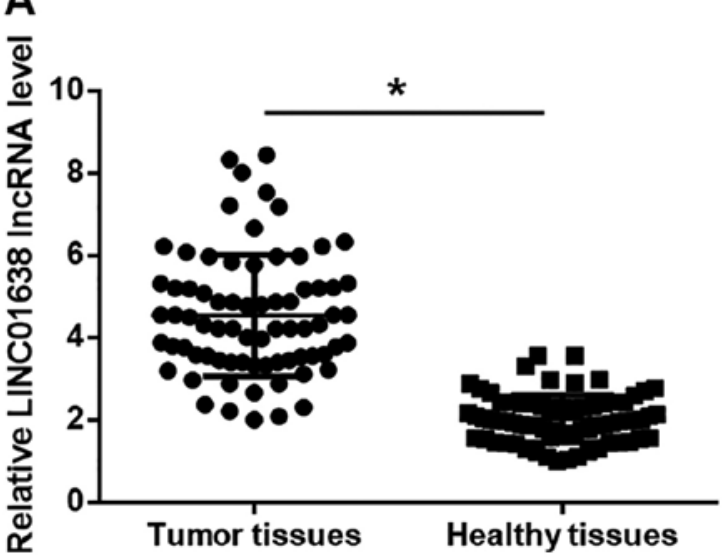

B

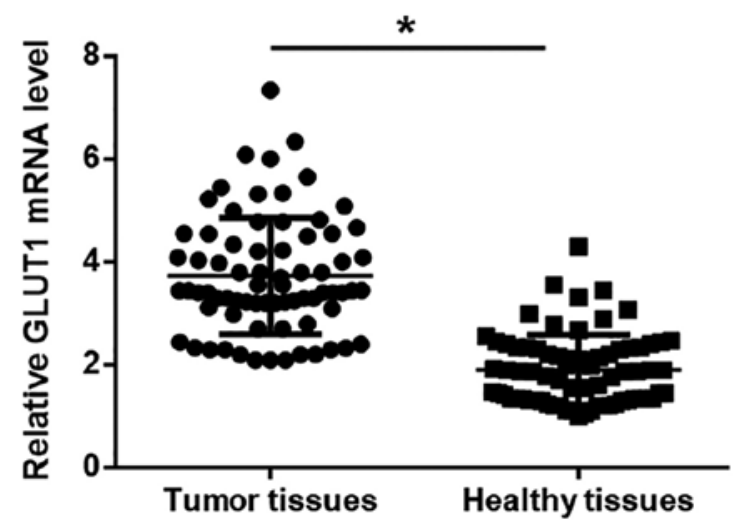

Figure 1. LINC01638 lncRNA and GLUT1 mRNA expression levels are upregulated in tumor tissues compared with adjacent healthy tissues in patients with HCC. Compared with adjacent healthy tissues, expression levels of (A) LINC01638 lncRNA and (B) GLUT1 mRNA were significantly higher in the tumor tissues of the 74 patients with $\mathrm{HCC}^{*} \mathrm{P}<0.05$. HCC, hepatocellular carcinoma; LINC01638, long intergenic non-protein coding RNA 1638; lncRNA, long non-coding RNA; GLUT1, glucose transporter 1.
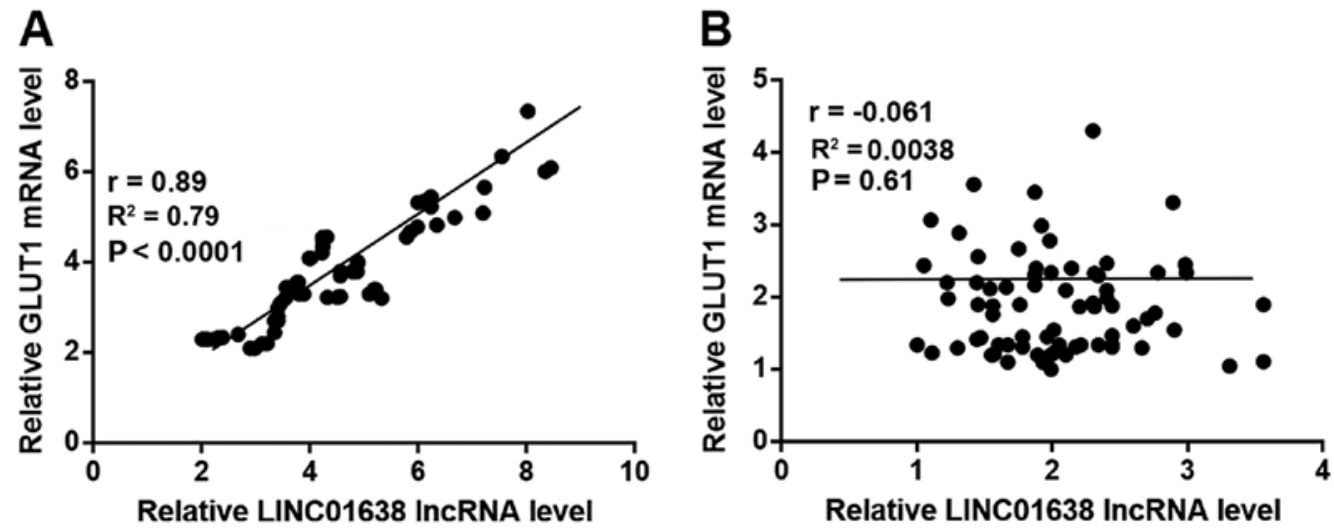

Figure 2. Expression levels of LINC01638 lncRNA and GLUT1 are positively correlated in tumor tissues, but not in adjacent healthy tissues. Expression levels of LINC01638 lncRNA and GLUT1 were significantly and positively correlated in (A) tumor tissues; (B) however, there was no correlation in adjacent healthy tissues. GLUT1, glucose transporter 1; LINC01638, long intergenic non-protein coding RNA 1638; lncRNA, long non-coding RNA.

deviation was calculated. All statistical analysis was performed using SPSS 19.0 software (IBM Corp., Armonk, NY, USA). Correlations between levels of LINC01638 1ncRNA and GLUT1 in tumor tissues and adjacent healthy tissues were performed by Pearson's correlation coefficient. Comparisons of levels of LINC01638 1ncRNA and GLUT1 between tumor tissue and adjacent healthy tissues were performed by paired t-test. Comparisons among three groups were performed by one-way ANOVA followed by Tukey's post hoc test. $\mathrm{P}<0.05$ was considered to indicate a statistically significant difference.

\section{Results}

LINC01638 IncRNA and GLUT1 mRNA are upregulated in tumor tissues compared with adjacent healthy tissues of patients with HCC. Expression levels of LINC01638 lncRNA and GLUT1 mRNA in tumor tissues and adjacent healthy tissues of 74 HCC patients were detected by RT-qPCR. Compared with adjacent healthy tissues, expression levels of LINC01638 lncRNA (Fig. 1A) and GLUT1 mRNA (Fig. 1B) were significantly higher in tumor tissues $(\mathrm{P}<0.05)$.
Expression levels of LINC01638 IncRNA and GLUT1 are positively correlated in tumor tissues. Correlation between expression levels of LINC01638 lncRNA and GLUT1 in tumor tissues and adjacent healthy tissues were performed by Pearson's correlation coefficient. The expression levels of LINC01638 lncRNA and GLUT1 were significantly and positively correlated in tumor tissues (Fig. 2A); however, there was no significant correlation between expression levels of LINC01638 lncRNA and GLUT1 in adjacent healthy tissues (Fig. 2B).

LINC01638 IncRNA and GLUT1 promotes glucose uptake in HCC cells. Glucose uptake assay indicated that, compared with the control and the negative control groups, overexpression of LINC01638 lncRNA and GLUT1 led to a significant increase in glucose uptake (Fig. 3A), while LINC01638 lncRNA and GLUT1-knockdown led to a significant inhibition in glucose uptake (Fig. 3B) in SNU-398 and SNU-182 cells $(\mathrm{P}<0.05)$.

LINC01638 IncRNA is a potential activator of GLUT1 in HCC cells. Compared with the control and the negative 

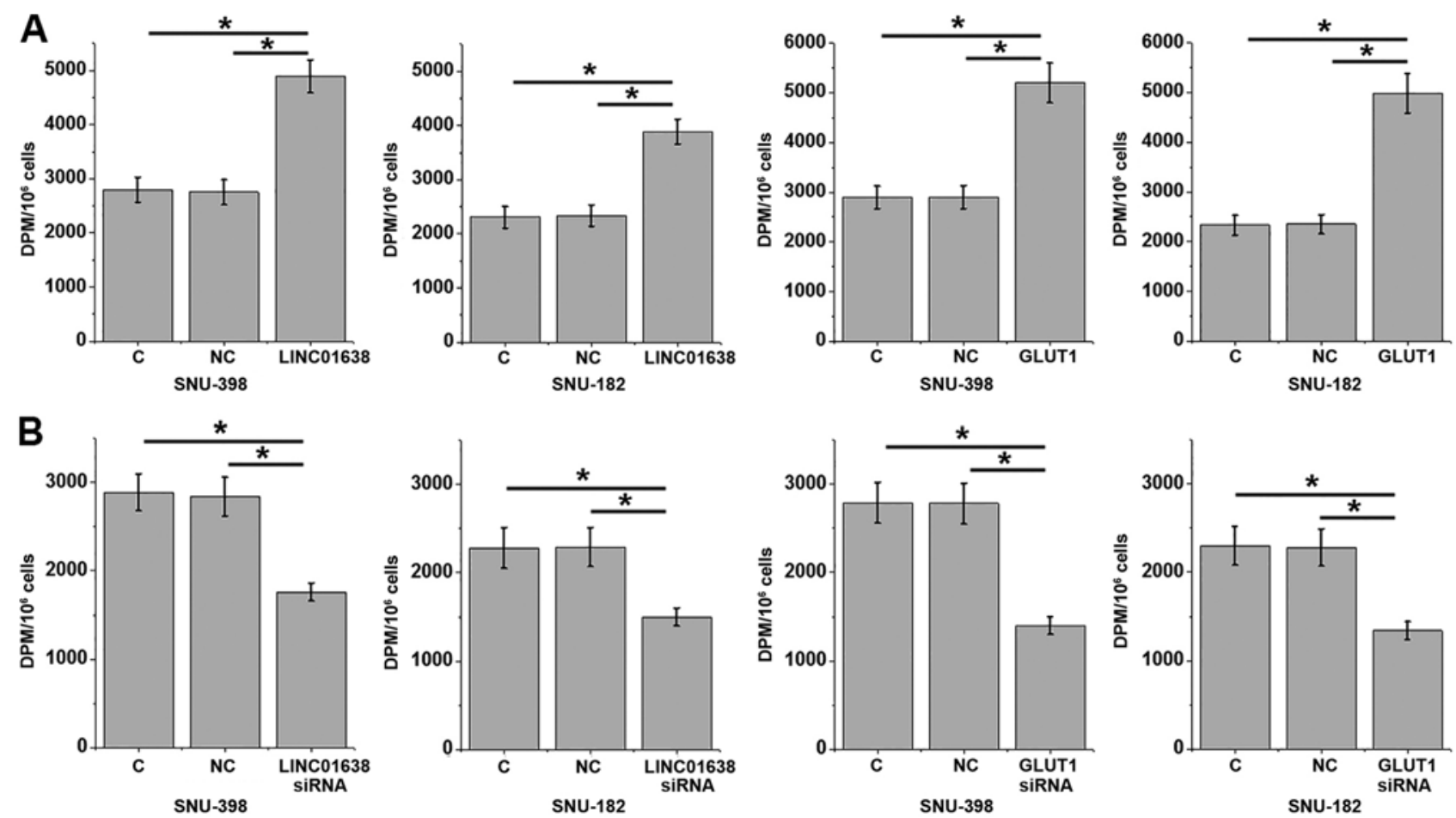

Figure 3. LINC01638 1ncRNA and GLUT1 promotes glucose uptake in hepatocellular carcinoma cells. (A) Overexpression of LINC01638 1ncRNA and GLUT1 led to significantly promoted glucose uptake, while (B) LINC01638 lncRNA and GLUT1-knockdown led to significantly inhibited glucose uptake in SNU-398 and SNU-182 cells. " $\mathrm{P}<0.05$. lncRNA, long non-coding RNA; DPM, disintegrations per minute; C, control cells; NC, negative control siRNA or empty vector; LINC01638, long intergenic non-protein coding RNA 1638; GLUT1, glucose transporter 1; siRNA, small interfering RNA.

control groups, overexpression of LINC01638 lncRNA led to significantly upregulated GLUT1 expression in SNU-398 and SNU-182 cells (Fig. 4A; P<0.05). By contrast, GLUT1 overexpression did not significantly affect LINC01638 lncRNA expression in SNU-398 and SNU-182 cells (Fig. 4B).

LINC01638 IncRNA promotes the proliferation of HCC cells through GLUT1. Compared with the control and the negative control groups, overexpression of LINC01638 lncRNA and GLUT1 led to increased proliferation, while LINC01638 lncRNA and GLUT1 siRNA silencing led to inhibited proliferation of HCC cells (Fig. 5). In addition, co-transfection experiments revealed that GLUT1 siRNA silencing attenuated the enhancing effects of LINC01638 lncRNA overexpression on cancer cell proliferation (Fig. 5).

\section{Discussion}

LINC01638 is a recently identified lncRNA with a function only characterized in breast cancer (13). To the best of our knowledge, the involvement of LINC01638 lncRNA in other types of cancer remains unknown. In the present study, LINC01638 IncRNA was upregulated in HCC, and the upregulation of LINC01638 lncRNA promoted glucose uptake in cancer cells and accelerated cancer cell proliferation. It was also indicated that the actions of LINC01638 lncRNA in HCC are likely mediated by the upregulation of GLUT1.

GLUT1, as a key player in cancer biology, is usually upregulated during the development of different types of cancer, including HCC (16). Consistent with previous studies, the present study also indicated that GLUT1 was upregulated in HCC tissues compared with adjacent healthy tissues. The upregulation of GLUT1 promotes cancer cell glucose uptake and affects cancer cell behaviors, including proliferation (17). In accordance with the aforementioned, the present study also demonstrated that GLUT1 was a positive regulator of glucose uptake in HCC cells and HCC cell proliferation. These data further confirmed the oncogenic role of GLUT1 in HCC.

A large set of lncRNAs are differentially expressed during the development and progression of HCC (18). In the present study the functionality of LINC01638 IncRNA was characterized, and LINC01638 also demonstrated to be upregulated in HCC. IncRNAs have key roles in glucose metabolism (19). In the present study, LINC01638 IncRNA was shown to promote glucose uptake in HCC cells, demonstrating the regulatory role of LINC01638 lncRNA in cancer cell energy metabolism. In addition, this study also suggested that LINC01638 lncRNA is a positive regulator of $\mathrm{HCC}$ cell proliferation. Therefore, these data suggest that LINC01638 lncRNA may be an oncogene in HCC.

It has been frequently observed that GLUT1 has a role in cancer biology through interaction with lncRNAs (20). For example, IncRNAs regulate GLUT1-mediated glycolysis to mediate tumor metastasis (20). The present study indicated that LINC01638 IncRNA may be an upstream activator of GLUT1 in HCC cells. In addition, the upregulation of GLUT1 by LINC01638 lncRNA may be involved in the regulation of HCC cell proliferation. However, the data also suggested that the interaction between LINC01638 lncRNA and GLUT1 is likely indirect, due to the significant correlation between LINC01638 1ncRNA and GLUT1 only in tumor tissues, without correlation in adjacent healthy tissues. 
A
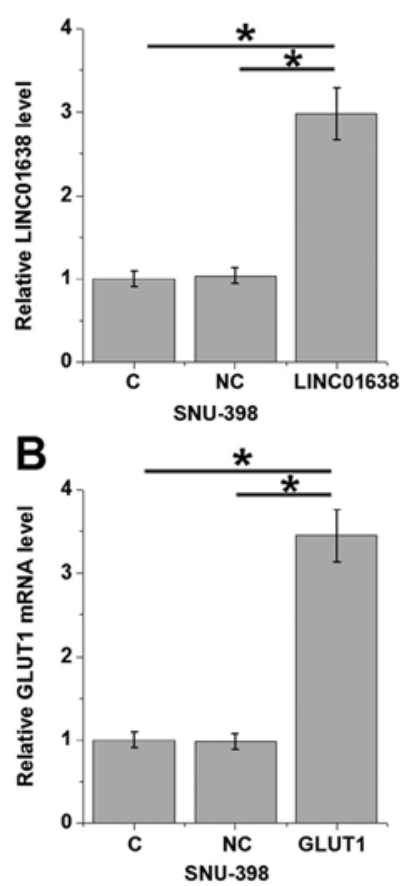

SNU-398
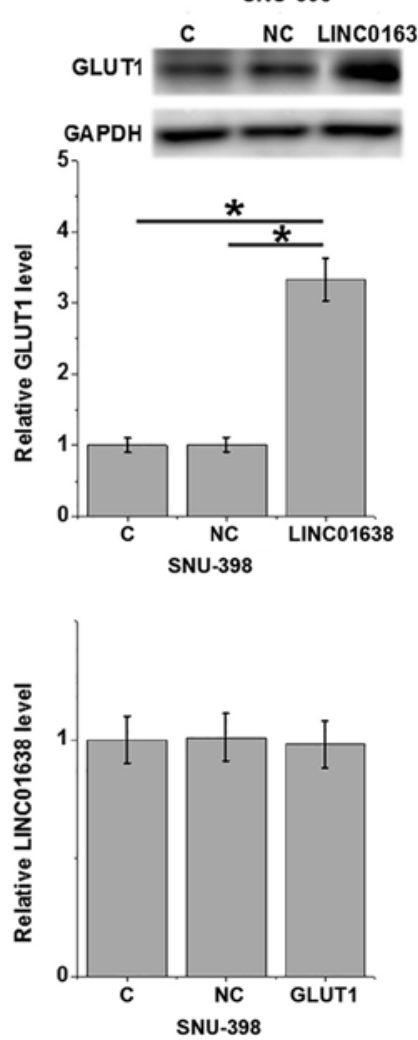
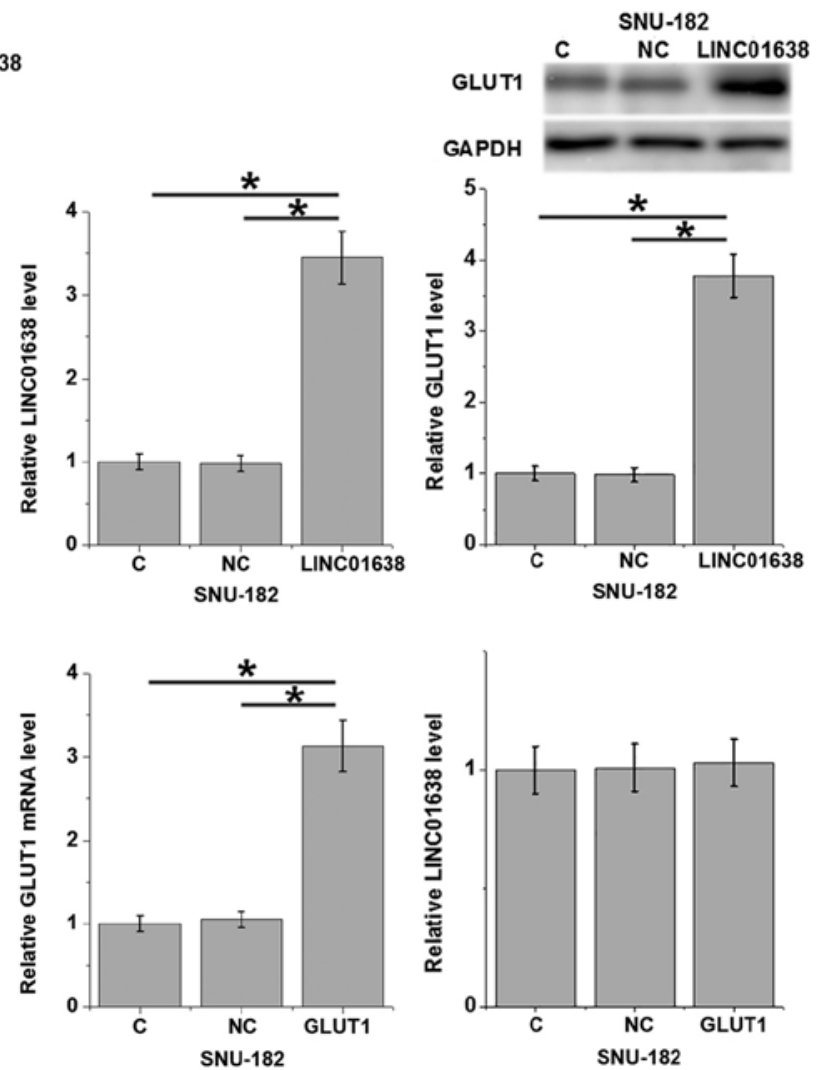

Figure 4. LINC01638 lncRNA is a potential activator of GLUT1 in hepatocellular carcinoma cells. (A) Overexpression of LINC01638 lncRNA led to significantly upregulated GLUT1 expression in SNU-398 and SNU-182 cells. (B) In contrast, GLUT1 overexpression did not significantly affect LINC01638 lncRNA expression in SNU-398 and SNU-182 cells. " $\mathrm{P}<0.05$. IncRNA, long non-coding RNA; LINC01638, long intergenic non-protein coding RNA 1638; C, control cells; NC, negative control empty vector; GLUT1, glucose transporter 1.

A
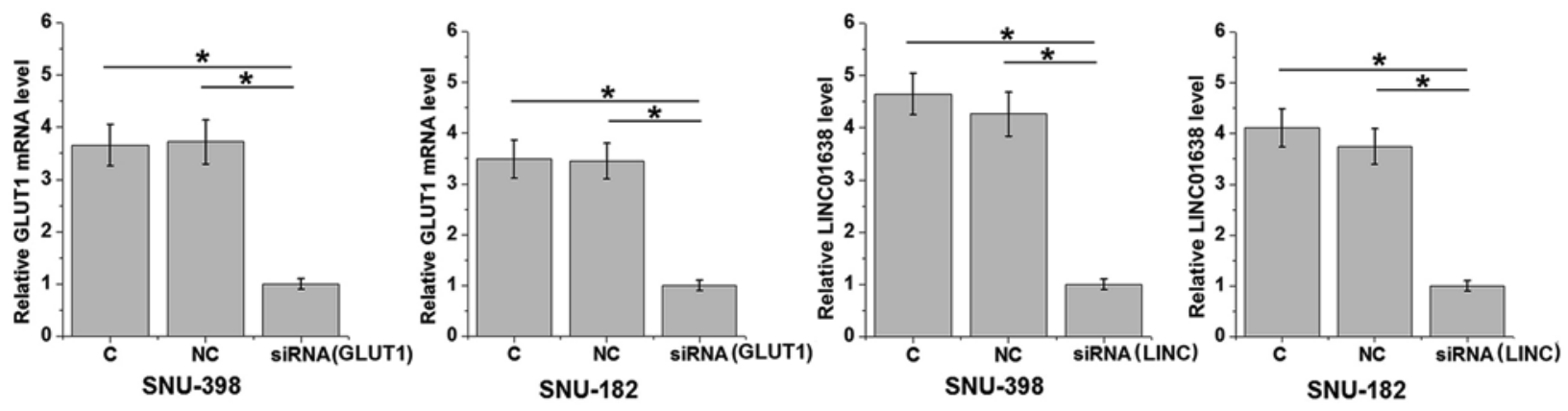

B
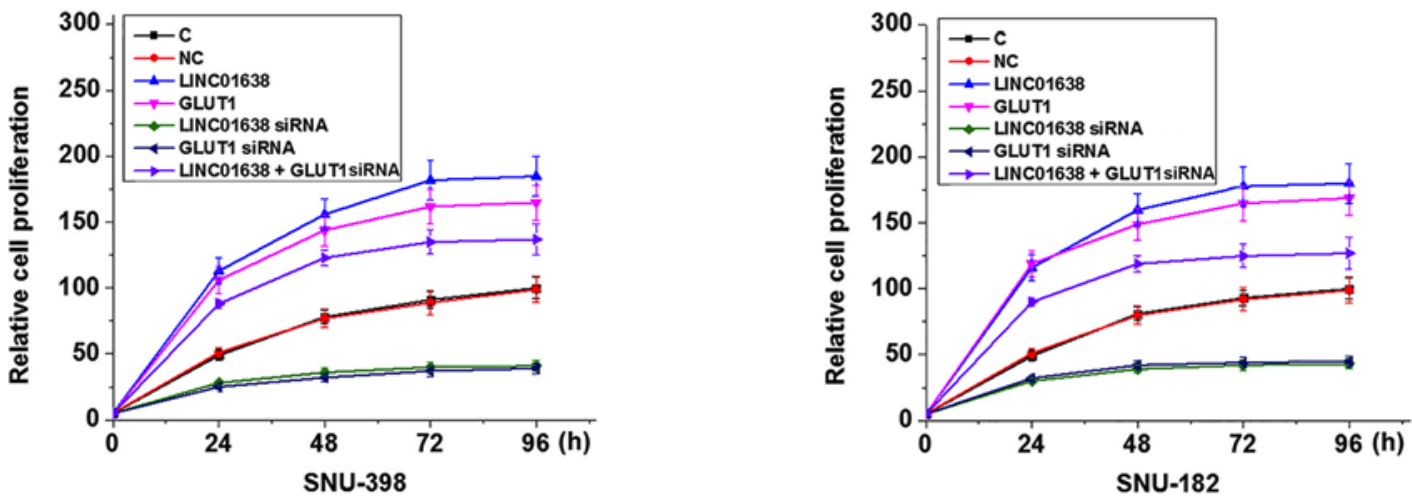

Figure 5. LINC01638 incRNA promotes the proliferation of HCC cells through GLUT1. (A) GLUT1 siRNA and LINC01638 siRNA were used to knockdown GLUT1 and LINC01638. (B) Overexpression of LINC01638 lncRNA and GLUT1 led a significant promotion of proliferation, while LINC01638 lncRNA and GLUT1-knockdown led to a significant inhibition of proliferation of HCC cells. In addition, GLUT1 knockdown attenuated the enhancing effects of LINC01638 lncRNA overexpression on cancer cell proliferation. "P<0.05. IncRNA, long non-coding RNA; HCC, hepatocellular carcinoma; GLUT1, glucose transporter 1; C, control cells; NC, negative control siRNA; siRNA, small interfering RNA; LINC01638, long intergenic non-protein coding RNA 1638. 
The present study used two cell lines derived from patients with $\mathrm{HCC}$ with hepatitis B virus (HBV) infection. Preliminary data indicated that LINC01638 expression was not affected by $\mathrm{HBV}$ and hepatitis $\mathrm{C}$ virus (HCV) infections (data not shown). This suggests that LINC01638 may participate in HCC through a HBV or HCV-independent pathway. Therefore, these two cell lines are appropriate for this study. In conclusion, LINC01638 lncRNA may be an oncogenic IncRNA in HCC. LINC01638 lncRNA may promote HCC by upregulating glucose uptake and promoting GLUT1 expression.

\section{Acknowledgements}

Not applicable.

\section{Funding}

Not applicable.

\section{Availability of data and materials}

The datasets used and/or analyzed during the present study are available from the author on reasonable request.

\section{Authors' contributions}

$\mathrm{HW}$ designed the experiments. XC and LW performed the experiments and collected the data. HW drafted the manuscript. All authors approved the manuscript.

\section{Ethics approval and consent to participate}

The present study was approved by The Ethics Committee of Sixth People's Hospital of Qingdao (Qingdao, China).

\section{Patient consent for publication}

Patients provided informed consent for publication of the present study.

\section{Competing interests}

The authors declare that they have no competing interests.

\section{References}

1. Torre LA, Bray F, Siegel RL, Ferlay J, Lortet-Tieulent J and Jemal A: Global cancer statistics, 2012. CA Cancer J Clin 65: $87-108,2015$.

2. Zhang Y, Ren JS, Shi JF, Li N, Wang YT, Qu C, Zhang Y and Dai M: International trends in primary liver cancer incidence from 1973 to 2007. BMC Cancer 15: 94, 2015.
3. Bruix J, Han KH, Gores G, Llovet JM and Mazzaferro V: Liver cancer: Approaching a personalized care. J Hepatol 62 (1 Suppl): S144-S156, 2015.

4. Momin BR, Pinheiro PS, Carreira H, Li C and Weir HK: Liver cancer survival in the United States by race and stage (2001-2009): Findings from the CONCORD-2 study. Cancer 123 (Suppl 24): S5059-S5078, 2017.

5. Zeng H, Zheng R, Guo Y, Zhang S, Zou X, Wang N, Zhang L, Tang J, Chen J, Wei K, et al: Cancer survival in China, 2003-2005: A population-based study. Int J Cancer 136: 1921-1930, 2015.

6. Lee MH, Kim EJ, Lee H, Kim HM, Chang MJ, Park SY, Hong KS, Kim JS and Sessler JL: Liposomal texaphyrin theranostics for metastatic liver cancer. J Am Chem Soc 138: 16380-16387, 2016.

7. Moreno-Sánchez R, Marín-Hernández A, Saavedra E, Pardo JP, Ralph SJ and Rodríguez-Enríquez S: Who controls the ATP supply in cancer cells? Biochemistry lessons to understand cancer energy metabolism. Int J Biochem Cell Biol 50: 10-23, 2014.

8. Pattni BS, Jhaveri A, Dutta I, Baleja JD, Degterev A and Torchilin V: Targeting energy metabolism of cancer cells: Combined administration of NCL-240 and 2-DG. Int J Pharm 532: 149-156, 2017.

9. Olson AL and Pessin JE: Structure, function, and regulation of the mammalian facilitative glucose transporter gene family. Annu Rev Nutr 16: 235-256, 1996.

10. Gwak HR, Haegeman G, Tsang BK and Song YS: Cancer-specific interruption of glucose metabolism by resveratrol is mediated through inhibition of Akt/GLUT1 axis in ovarian cancer cells. Mol Carcinog 54: 1529-1540, 2015.

11. Liu X and Gan B: lncRNA NBR2 modulates cancer cell sensitivity to phenformin through GLUT1. Cell Cycle 15: 3471-3481, 2016.

12. Spizzo R, Almeida MI, Colombatti A and Calin GA: Long non-coding RNAs and cancer: A new frontier of translational research? Oncogene 31: 4577-4587, 2012.

13. Luo L, Tang H, Ling L, Li N, Jia X, Zhang Z, Wang X, Shi L, Yin J, Qiu N, et al: LINC01638 lncRNA activates MTDH-Twist1 signaling by preventing SPOP-mediated c-Myc degradation in triple-negative breast cancer. Oncogene 37: 6166-6179, 2018.

14. Chun YS, Pawlik TM and Vauthey JN: 8th edition of the AJCC cancer staging manual: Pancreas and hepatobiliary cancers. Ann Surg Oncol 25: 845-847, 2018.

15. Livak KJ and Schmittgen TD: Analysis of relative gene expression data using real-time quantitative PCR and the 2(-Delta Delta $\mathrm{C}(\mathrm{T})$ ) method. Methods 25: 402-408, 2001.

16. Amann T, Maegdefrau U, Hartmann A, Agaimy A, Marienhagen J, Weiss TS, Stoeltzing O, Warnecke C, Schölmerich J, Oefner PJ, et al: GLUT1 expression is increased in hepatocellular carcinoma and promotes tumorigenesis. Am J Pathol 174: 1544-1552, 2009.

17. Sawayama H, Iwatsuki M, Yoshida N, Baba Y, Sakamoto $Y$, Kinoshita K, Nakamura K, Kuroda D, Tsugio E, Toihata T, et al: Glucose transporter 1 is associated with proliferation and prognosis on esophageal squamous cell carcinoma. Cancer Res 77: 4407, 2017.

18. Zhu J, Liu S, Ye F, Shen Y, Tie Y, Zhu J, Jin Y, Zheng X, Wu Y and $\mathrm{Fu} \mathrm{H}$ : The long noncoding RNA expression profile of hepatocellular carcinoma identified by microarray analysis. PLoS One 9: e101707, 2014

19. Ruan X: Long non-coding RNA central of glucose homeostasis. J Cell Biochem 117: 1061-1065, 2016.

20. Wang Y, Zhang X, Wang Z, Hu Q, Wu J, Li Y, Ren X, Wu T, Tao X, Chen X, et al: lncRNA-p23154 promotes the invasion-metastasis potential of oral squamous cell carcinoma by regulating Glut1-mediated glycolysis. Cancer Lett 434: 172-183, 2018. 